\title{
Novel splice site IDUA gene mutation in Tunisian pedigrees with hurler syndrome
}

\author{
Latifa Chkioua ${ }^{1,4^{*}}$, Hela Boudabous², Ibtissem Jaballi', Oussama Grissa', Hadhami Ben Turkia², Neji Tebib² \\ and Sandrine Laradi ${ }^{3}$
}

\begin{abstract}
Background: The mucopolysaccharidosis type I (MPS I) is a lysosomal storage disease resulting from the defective activity of the enzyme a-L-iduronidase (IDUA). The disease has three major clinical subtypes (severe Hurler syndrome, intermediate Hurler-Scheie syndrome and attenuated Scheie syndrome). We aim to identify the genetic variants in MPS I patients and to investigate the effect of the novel splice site mutation on splicing of IDUA- mRNA variability using bioinformatics tools.
\end{abstract}

Methods: The IDUA mutations were determined in four MPS I patients from four families from Northern Tunisia, by amplifying and sequencing each of the IDUA exons and intron-exon junctions.

Results: One novel splice site IDUA mutation, c.1650 + 1G > T in intron 11 and two previously reported mutations, p.A75T and p.R555H, were detected. The patients in families 1 and 2 who have the Hurler phenotype were homozygotes for the novel splice site mutation c. $1650+1 G>T$. The patient in family 3, who also had the Hurler phenotype, was a compound heterozygote for the novel splice site mutation c.1650 + 1G > T and for the previously reported missense mutation p.A75T. The patient in family 4 who had the Hurler-Scheie phenotype was a compound heterozygote for the novel splice site mutation c.1650 +1G > T and for the previously reported missense mutation p.R555H. In addition, four known IDUA polymorphisms were identified. Bioinformatics tools allowed us to associate the variant c.1650 + 1G > T with the severe clinical phenotype of MPS I. This variant affects the essential nucleotide +1 ( $G$ to $T$ ) of the donor splice site of IDUA intron 11. The G $>$ T in intron 11 leads to wild type donor site broken with minus $19.97 \%$ value compared to normal value with $0 \%$, hence the new splice site acceptor has plus 5.59\%.

Conclusions: The present findings indicate that the identified mutations facilitate the accurate carrier detection (genetic counseling of at-risk relatives) and the molecular prenatal diagnosis in Tunisia.

Keywords: Mucopolysaccharidosis type I, a-L-iduronidase, Splice site mutation, Homozygous, Compound heterozygote

\section{Background}

Mucopolysaccharidosis type I (MPS I) is an autosomal recessive lysosomal storage disorder caused by the deficient activity of the enzyme $\alpha$-L-iduronidase (IDUA, EC 3.2.1.76). This glycosidase is required for the hydrolysis of $\alpha$ L-iduronide residues of dermatan sulphate and heparan sulphate [1].

MPS I has three major clinical subtypes among which the severe Hurler syndrome (MPS IH; 607014). It is

\footnotetext{
* Correspondence: chkioualatifa2002@yahoo.fr

${ }^{1}$ Faculty of pharmacy, University of Monastir, 5000 Monastir, Tunisia

${ }^{4}$ Faculty of pharmacy of Monastir, University of Monastir, Avenue Avicenne,

5019 Monastir, Tunisia

Full list of author information is available at the end of the article
}

characterized by infantile onset, severe organomegaly and bone involvement, and mental retardation. The intermediate Hurler-Scheie syndrome (MPS IH/S; 607015) is characterized by onset in childhood, severe organomegaly and bone involvement, and usually limited, if any, neurological involvement. The attenuated Scheie syndrome (MPS IS; 607016) is characterized by later onset, visceral and bone disease, and neurological developmental delay [1].

The IDUA gene has $19 \mathrm{~kb}$ in length, containing 14 exons and 13 introns. It is mapped on the short arm of chromosome 4 at region p16.3 [2] being transcribed into a $2.3 \mathrm{~kb}$ cDNA, which encodes a 653-residue glycopeptide [3]. 
To date, more than 201 mutations and 32 polymorphisms have been identified [Human Gene Mutation Database] (http://www.hgmd.org; 2017). Previous mutations include 113 missense/nonsense, 33 splicing, 31 small deletions, 15 small insertions, 4 gross deletions and 3 complex rearrangements. Genetic testing MPS I patients is useful for the identification of specific genotypes, genotype-phenotype correlations and also for prenatal diagnosis.

The splice site mutations are DNA sequence changes that alter or abolish correct mRNA splicing during the process of precursor mRNA maturation. The modification in the consensus sequence, known as splice-donor and splice-acceptor sequences, which surround each exon may lead to: exon skipping, cryptic splice site activation, creation of a pseudo-exon within an intron and intron retention [4]. Hence some splice site mutations do not abolish completely the wild-type transcript expression, which may lead to less severe phenotypes [5]. In our study, we have analyzed the novel splice donor site c. $1650+1 \mathrm{G}>\mathrm{T}$ in intron 11 using bioinformatics tools to determine the impact of this variant in MPS I phenotypic expression.

\section{Methods}

\section{Ethics statements}

Written informed consent was obtained and signed by all studied families after a full explanation of this study, which was approved by the local ethic committees for scientific research of the La Rabta Hospital Tunis, Tunisia. Additional informed consent was obtained from all patients for whom identifying information is included in this study. All procedures were in accordance with the ethical standards of the responsible committee on human experimentation (institutional and national) and with the Helsinki Declaration.

\section{Study populations}

This is a series of four patients (P1, P2, P3 and P4) with MPS I disease aged 2-5 years who were recruited in the pediatric department of La Rabta Hospital in Tunisia. Among the four explored MPS I families, three (family 1, family 2 and family 4) are related as second cousins. The MPS I patients had a clinical diagnosis of Hurler syndrome which was further confirmed with biological analysis by demonstrating a high excretion of GAGs in the urine and a deficiency in $\alpha$-L-iduronidase activity in leukocytes. The parents and other family members of each studied family were investigated in order to create a clearer profile of the disease's transmission to facilitate prenatal diagnosis and counseling for families at risk in Tunisia.

In this study based on clinical manifestations of MPS I patients, an analysis of urine glycosaminoglycans (GAGs) was done in first intention but this screening requires a differential diagnosis with the Hunter syndrome for which we obtained the same GAG profile.

\section{Biochemical diagnosis}

The diagnosis of these diseases was based on the following approach after a clinical and paraclinical suspicion.

\section{Quantitative and qualitative analysis of total urinary glycosaminoglycans}

Study of urinary glycosaminoglycans was performed first. Urinary GAGs were quantified using a dimethylmethylene blue test (DMB) [6]. The quantity of DMB bound to sulfated glycosaminoglycans was measured via spectrophotometry at wavelength of $656 \mathrm{~nm}$. Electrophoresis on cellulose acetate plate was performed to identify which type of GAGs is present in excess (e.g., dermatan sulphate, heparan sulphate, keratan sulphate). Discontinuous electrophoresis on cellulose acetate plate separated the different GAGs based on their charge and differential solubility in ethanol, and the mucopolysaccharides were visualized by staining with alcian blue [6].

\section{Enzyme analysis}

Enzyme analysis for $\alpha$-L-iduronidase (MPSI, EC 3.2.1.76) was performed in sonicated leukocytes pellets as described using the 4-methylumbelliferyl- $\alpha$-L-iduronide [7].

\section{Molecular analysis and DNA sequencing analysis}

We analyzed the IDUA gene of 4 MPS I patients from Northern Tunisia using PCR, PCR-based restriction fragment length polymorphism (RFLP) and direct sequencing methods.

Genomic DNA was isolated from venous blood by the phenol/chloroform procedure according to standard protocols as described previously [8]. All the exons and flanking intron/exon junctions of the IDUA gene were amplified and sequenced. For patients with a family history of known or suspected pathogenic mutations or for the indexed cases of parents, the targeted DNA locus was analyzed.

PCR reaction consisted of 50 ng of DNA,1 X HotStarTaq buffer (Qiagen, Paris, France), $2 \mathrm{mM} \mathrm{MgCl}$, $200 \mu \mathrm{M}$ of each dNTP, 10 pmol of each primer, $2.5 \mathrm{U}$ of HotStarTaq (Qiagen, Paris, France) and $1 \mathrm{X}$ Q solution. The final reaction volume was $18 \mu \mathrm{l}$. Thermal PCR profile consisted of an initial denaturation at $95{ }^{\circ} \mathrm{C}$ for $15 \mathrm{~min}, 35$ cycles of denaturation at $94{ }^{\circ} \mathrm{C}$ for $30 \mathrm{~s}$, annealing at $68{ }^{\circ} \mathrm{C}$ for 30 s and extension at $72{ }^{\circ} \mathrm{C}$ for $30 \mathrm{~s}$ followed by a final extension step at $72{ }^{\circ} \mathrm{C}$ for $10 \mathrm{~min}$. PCR products were resolved in $2 \%$ agarose gel and were visualized under UV light.

\section{In silico predictions}

The splice site mutation was located in the defined splice site consensus sequences which were (C/ A)AG|gt(a/g)agt and cag|G, for the donor splice site and the acceptor splice site, respectively [9]. 
We have analyzed the splice site mutation in intron 11 of IDUA gene by using the bioinformatics tools: Human Splicing Finder (HSF) version 2.4 [10] (http:// www.umd.be/HSF/) which includes several matrices to analyze splice site and Exonic Splicing Enhancer (ESE) finder predictions (http://krainer01.cshl.edu/cgi-bin/ tools/ESE3/esefinder.cgi?process=home) to examine the conservation of ESE motifs.

\section{Results}

\section{Clinical features and biochemical analysis}

The clinical features of each patient are presented in Table 1.

Phenotypic analysis confirmed the diagnosis of all the MPS IH studied patients. Indeed, for all the studied patients, the electrophoresis on cellulose acetate plate of GAGs showed the presence of heparan sulphate (HS) and dermatan sulphate (DS), an abnormal band, compared to the control case, in addition to an abnormal band of HS in MPSIII patient (Fig. 1).

IDUA activity in MPS IH patients ranged from 0.00 to $0.044 \mu \mathrm{Kat} / \mathrm{Kg}$ protein.

\section{IDUA mutation analysis}

Clinical and identified genotypes of studied patients are summarized in Table 1.

As a result of DNA sequencing analysis and RFLP-PCR, one novel and two previously reported mutations were identified in this study including: two missense mutations p.A75T and p.R555H, and one novel splice site mutation c. $1650+1 \mathrm{G}>\mathrm{T}$ (Table 1).

Patients 1 and 2 from families 1, and 2 (Fig. 2) were all homozygous for a novel $\mathrm{G}$ to $\mathrm{T}$ transition in the conserved $5^{\prime}$ splice donor site of IDUA intron 11 (CAGGc > CAGTc) (Table 2). The splice site mutation obliterated a Cac8I restriction enzyme site. The amplicon of exon 11 from genomic DNA and its digestion with Cac8I resulted in three fragments $(66,101$ and $378 \mathrm{bp})$ in the patients with the splice site mutation, instead of the four fragments (23, 66, 101 and $356 \mathrm{bp}$ ) observed in normal individuals (data not shown).

Patient 3 was a compound heterozygote for a $\mathrm{G}$ to $\mathrm{A}$ transition in exon 2 (Fig. 2) predicting an Alanine to a Threonine missense mutation (p.A75T) and to a novel splice site mutation c. $1650+1 \mathrm{G}>\mathrm{T}$. The p.A75T missense mutation created a MSLI restriction enzyme site. The MSLI digestion of the amplicon of exon 2 from genomic DNA resulted in two fragments (166 and $139 \mathrm{bp}$ ) in the patient with the missense mutation, instead of the one fragment (305 bp) observed in normal individuals (data not shown).

Patient 4 was also a compound heterozygote for a G to A transition in exon 11 (Fig. 2) predicting an Arginine to a Histidine missense mutation (p.R555H) and to the novel splice site mutation c. $1650+1 \mathrm{G}>\mathrm{T}$.

In addition, four previously reported polymorphisms in exon 11 of IDUA gene were identified in the IDUA patients and their parents: rs141046991, rs773947412, rs759123051, rs773184536 (Table 1).

\section{Splice site mutation analysis}

The relative strength of the splicing sites obtained from the bioinformatics tool is given as a consensual value (CVs), which varies from 0 to 100 . Therefore, the effect of a splice site mutation depends on the $\mathrm{CVs}$ value. Splice sites with CVs over 80 are solid splicing sites, but splicing sites with CVs ranging from 65 to 70 are weak sites because only a few of these sites are active [11].

The splice site mutation leads to the use of cryptic sites thus the most cryptic splice sites are to be located +/- $100 \mathrm{bp}$ on each side of the exon-intron boundary. We have analyzed this region for the presence of potential splice sites using HSF version 2.4 (http:// www.umd.be/HSF/) in the case of c. $1650+1 \mathrm{G}>\mathrm{T}$ mutation and we have found that the abolition of the wild type donor splice site, with minus $19.97 \%$ value, and its substitution by a new splice acceptor site, with plus $5.59 \%$ value, leads to three possible conclusions (Fig. 3): firstly, the loss of exon 11; secondly, the retention of the part of the intron 11 sequence; and finally, the activation of an alternative cryptic splicing site in exon 11 giving a new size of exon 11 of about $135 \mathrm{bp}$ compared to the normal length of $304 \mathrm{bp}$ (Table 2).

The results using the Human Splicing Finder, showed that the wild phenotype has a donor splice site CVs close to 100 , thus this new splice site could be strong and functional enough to justify the mutant phenotype which presented a new splice acceptor site with a CV at about 80 which would justify the new isoform of mRNA in patients (Table 2).

\section{Discussion}

This work was conducted as a straight continuation of studies carried out in other Tunisian MPS I patients and their families [12-15]. In this cohort we were interested in patients presenting a severe phenotype of MPS I. All MPS IH patients have the splice site c. $1650+1 \mathrm{G}>\mathrm{T}$ mutant allele in homozygous and/or heterozygous forms.

Our study showed that all studied patients were from different regions within the Northern part of Tunisia: Tunis (Oued Ellil) and Nabeul (Korba and Elmaamoura) that are over $60 \mathrm{~km}$ away from each other; however, three families (P1, P2 and P4) were known to be related.

Patients 1 and 2 (P1 and P2) who developed a severe form of MPS I were homozygotes for the novel splice site mutation c. $1650+1 \mathrm{G}>\mathrm{T}$. 


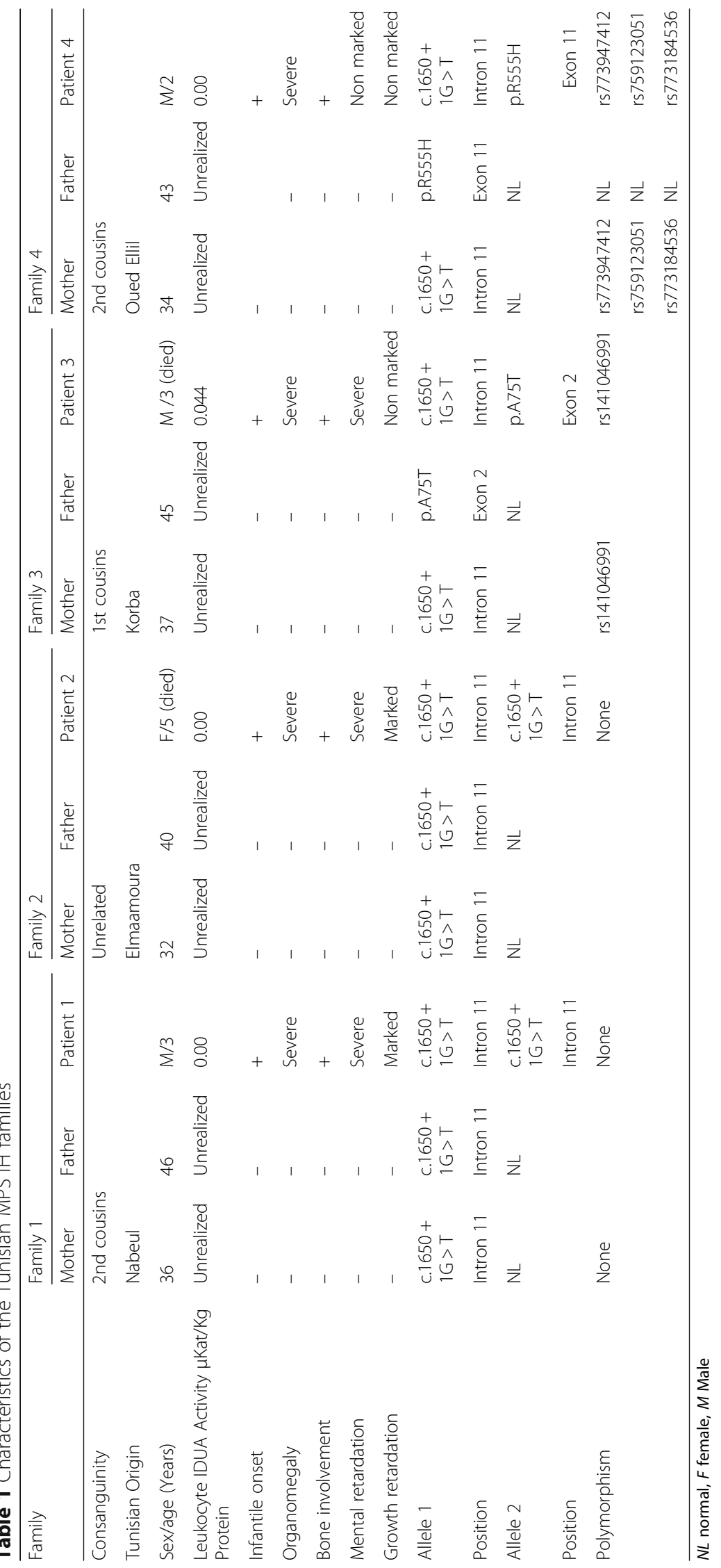




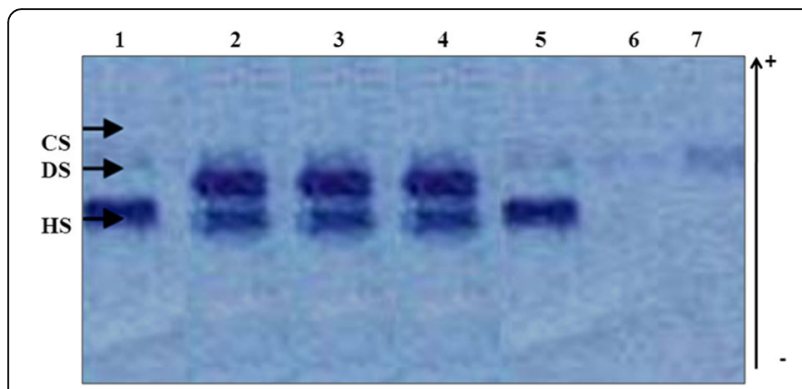

Fig. 1 MPS I electrophoresis profile on a cellulose acetate plate of the urinary GAGs. 1 and 5: MPS III controls; 2,3 and 4: MPS I or MPS II patients; 6 and 7: Normal controls. CS: chondroitin sulphate; DS: dermatan sulphate; HS: heparan sulphate

Our results about P1 and P2 using in silico predictions showed that the wild donor splice site and the mutant splice site have higher $\mathrm{CVs}$ and below 80 respectively.

A splice site mutation may cause activation of an alternative cryptic splice site, preferred to the use of the legitimate splice site. The $\mathrm{G}>\mathrm{T}$ mutation at the $5^{\prime}$-donor splice site of intron 11 presumably causes exon skipping, the loss of exon 11, and subsequently an aberrant polypeptide that is misfolded (http://rulai.cshl.edu/tools/ESE3) which should explain the severe phenotype of MPS I. In further studies, we may suggest to perform in vitro analysis in order to confirm the in silico predictions and therefore i) to study the impact of the splice site mutation during the process of precursor mRNA maturation, ii) to analyze the phenotypic expression of this disease.

Prenatal diagnosis was performed in family 1 . The fetus was found homozygous for the splice site mutation however the parents refused the interruption of pregnancy. Then, the patient 1 was included in the register of patients wishing to perform a bone marrow transplant but neither matching relative nor an unrelated matched donor has been found so far.

The P3 of family 3 does not present any relationship with the other investigated families but he lives in the same

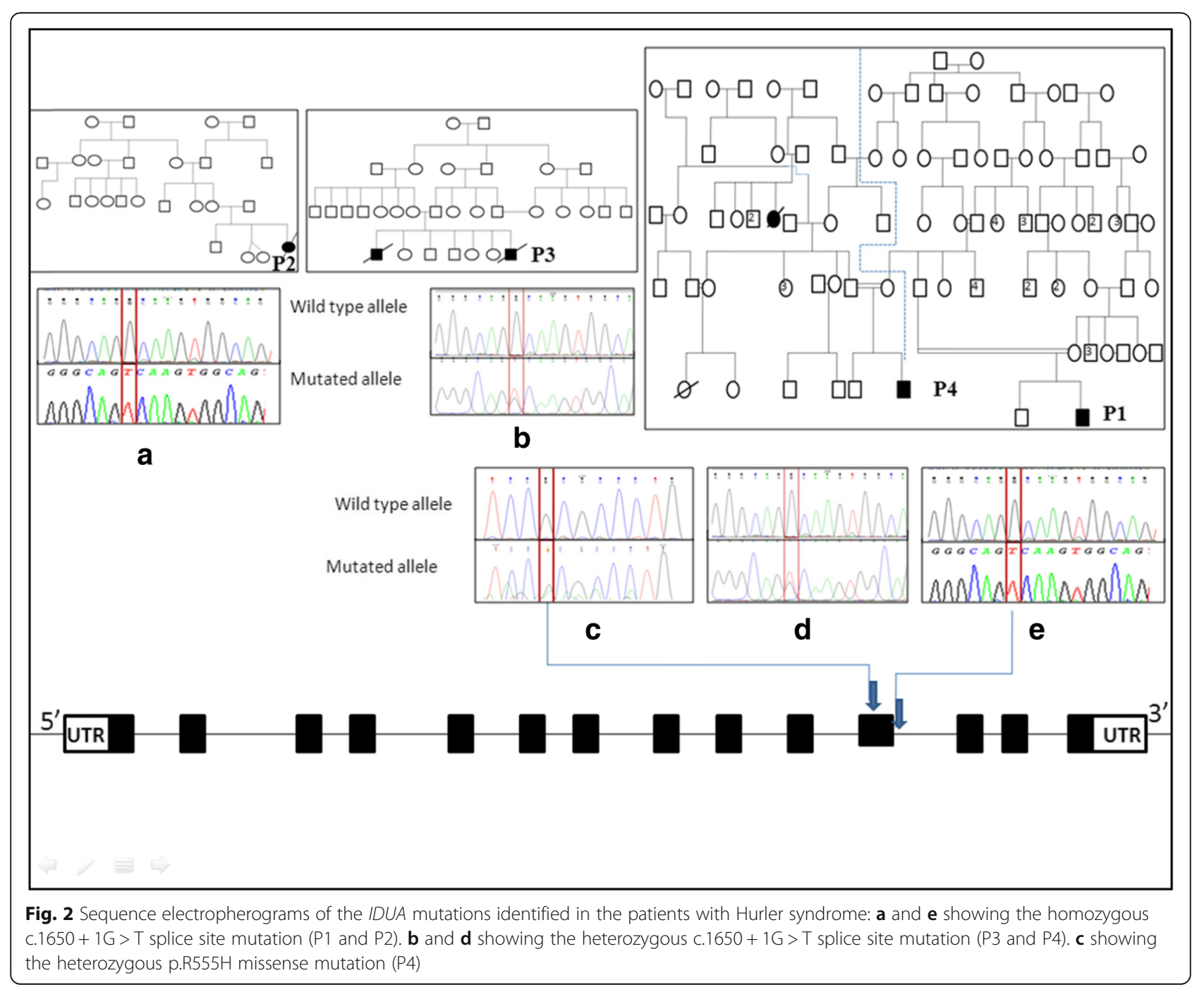


Table 2 Data findings: CVs and percentage variation of wild type and mutant sequences in intron 11 of IDUA gene (http:// www.umd.be/HSF/)

\begin{tabular}{lllllll}
\hline Splice site type & Motif & New splice site & $\begin{array}{l}\text { Wild type (WT) } \\
\text { CV (0-100) }\end{array}$ & $\begin{array}{l}\text { Mutant CV } \\
(0-100)\end{array}$ & $\begin{array}{l}\text { If cryptic site use, exon } \\
\text { length variation (bp) }\end{array}$ & Variation (\%) \\
\hline Acceptor & CCGCCCGGGCAGgC & ccgcccgggcagTC & 84.05 & 79.76 & NA & -5.1 \\
Acceptor & CGGGCAGgcaagtg & ccgggcagtcaagTG & 64.94 & 68.57 & NA & New site + 5.59 \\
Donor & CAGgcaagt & CAGtcaagt & 100 & 80.03 & 135 & WT site broken -19.97 \\
Acceptor & Ggcaagtggcagtc & gtcaagtggcagTC & 73.99 & 77.65 & NA & +4.95 \\
\hline
\end{tabular}

region of Tunisia. He was found to be a compound heterozygote for the novel splice site mutation and the previously reported missense mutation p.A75T. He developed severe features at an early age ( 9 months); this finding is in agreement with those reported in the literature [16]. The severe phenotype observed in this patient, results from the association of both mutated alleles: c. $1650+1 \mathrm{G}>\mathrm{T}$ and p.A75T . Thus, this new splice site appears to be strong and functional enough to justify the mutant phenotype observed in P3 which presents a new splice acceptor site with a CV at

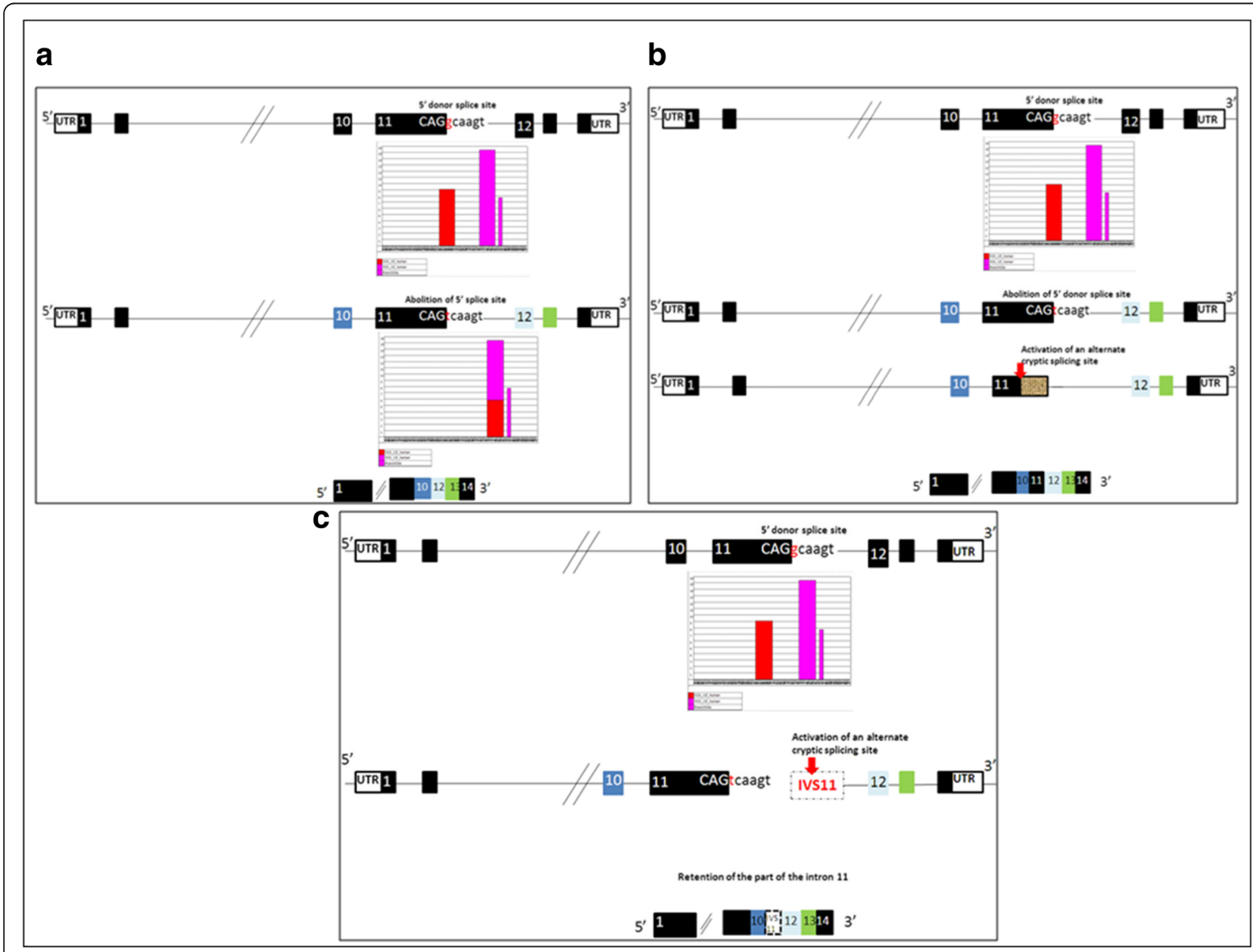

5SS_U2_human

3SS_U2_human

BranchSite

Fig. 3 Effects of IDUA splice site mutation. a Mutation c.1650 + 1G > T, which abolies a splice donor (GT) within intron 11, resulting in skipping of the exon 11. b Mutation c. $1650+1 \mathrm{G}>\mathrm{T}$, which creates an alternate cryptic splicing site, resulting in the deletion of 169 nucleotides at the end of the exon 11 giving a new size of exon 11 about 135 bp. c Mutation c.1650 + 1G > T which retains the part of the intron 11 
about 80 and which would justify the new isoform of mRNA in this patient. The p.A75T mutation has also an impact on phenotype manifestation. This genetic lesion was a non conservative mutation resulting from a change of a non polar Alanine to a polar Threonine at position 75 of IDUA protein. The p.A75T mutation was described for the first time in a patient with a severe MPS I in North America [17]. Patient 3 died at the age of three and his brother, who developed the similar severe clinical phenotype died before our molecular investigation; he probably had the same genetic lesion as patient P3.

Family 4 appears to present a relationship with the other studied families (1 and 2), as second cousins. P4 developed early clinical manifestations at 9 months of age, e.g. dysmorphic facial appearance and hepatosplenomegaly, hence these features were presented in moderate form at this age. Patient 4 was a compound heterozygote for the novel splice site mutation and the previously reported missense mutation p.R555H. The molecular analysis of genomic DNA of parents confirmed the segregation of the mutants' alleles. His parents were heterozygous for the splice site mutation c. $1650+1 \mathrm{G}>\mathrm{T}$ and for the missense mutation p.R555H, respectively.

The p.R555H missense mutation in exon 11 occurred at a CpG dinucleotide, a hotspot for mutation [18], and resulted in a nonconservative transition of a basic Arginine to a neutral Histidine. Homozygous patients for the p.R555H mutation have been reported from European countries in patients with the severe phenotype.

The investigation of Tunisian patients suffering from Hurler disease allowed us to define a geographical distribution of the IDUA mutations. Thus, the missense mutation p.P533R was recurrent in MPS I patients of Southern Tunisia [11] whereas the splice site mutation c. $1650+1 \mathrm{G}>$ $\mathrm{T}$ seems to be frequent in Northern Tunisia. Neither of these mutations have been identified in a different territory from those in their regions of origin. It is noteworthy that in the Maghreb (Morocco, Tunisia) only the p.P533R-MPS I missense mutation has been identified so far $[10,11]$.

Unfortunately, some MPS I patients die before their investigations. This can be explained by the clinical difficulty and thus the delay of diagnosis of the MPS I inherited disease. The adverse socioeconomic conditions of those patients make the situation even more difficult.

\section{Conclusion}

The novel mutation expands the IDUA gene mutation spectrum and contributes to the recognition of its impact on phenotypic expression in MPS I patients. The geographical distribution of these most frequent mutations observed in MPS I patients- the novel splice site mutation c. $1650+1 \mathrm{G}>\mathrm{T}$ and the missense mutation p.P533R in Northern and Southern of Tunisia respectively- will be useful for the identification of the carrier.

\section{Abbreviations}

DMB: Dimethylmethylene blue test; GAGs: Glycosaminoglycans; HSF: Human Splicing Finder; IDUA: a-L-iduronidase; MPS IH: Mucopolysaccharidosis type IH; MPSI: Mucopolysaccharidosis type I; WT: Wild type

\section{Acknowledgements}

We thank all the families with MPS $I H$ for their participation in this study and the clinicians for their fruitful involvement in this work.

\section{Availability of data and materials}

Novel genetic data from this study has been deposited in the European Nucleotide Archive [Accession number: LT960372] (https://www.ebi.ac.uk/ ena/data/view/LT960372).

\section{Authors' contributions}

LC carried out all the experiments, the data analyses, and wrote the manuscript. $\mathrm{HB}$ and IJ supported the analysis and interpretation of the data. $\mathrm{OG}, \mathrm{HB}, \mathrm{NT}$ and SL revised the manuscript. All authors participated in writing the manuscript and approved the final version.

\section{Ethics approval and consent to participate}

The study was approved by the ethics committees for scientific research of the La Rabta Hospital Tunis, Tunisia; no reference number was issued.

\section{Consent for publication}

Written informed consent for publication of their clinical details and/or clinical images was obtained from the parents of each patient. Copies of the consent forms are available for review by the Editor of this journal.

\section{Competing interests}

The authors declare that they have no competing interests.

\section{Publisher's Note}

Springer Nature remains neutral with regard to jurisdictional claims in published maps and institutional affiliations.

\section{Author details}

${ }^{1}$ Faculty of pharmacy, University of Monastir, 5000 Monastir, Tunisia. ${ }^{2}$ La Rabta Hospital, 1007 Tunis, Tunisia. ${ }^{3}$ The Auvergne-Rhône-Alpes Regional Branch of the French National Blood System EFS/GIMAP-EA-3064, 42023 Saint Etienne, France. ${ }^{4}$ Faculty of pharmacy of Monastir, University of Monastir, Avenue Avicenne, 5019 Monastir, Tunisia.

Received: 11 September 2017 Accepted: 13 May 2018

Published online: 29 May 2018

\section{References}

1. Neufeld EF. The mucopolysaccharidoses. The metabolic and molecular bases of inherited disease; 2001. p. 3421-52.

2. Scott HS, Bunge S, Gal A, Clarke LA, Morris CP, Hopwood JJ. Molecular genetics of mucopolysaccharidosis type I: diagnostic, clinical, and biological implications. Hum Mutat. 1995;6:288-302.

3. Scott H, Anson D, Orsborn A, Nelson P, Clements P, Morris C, Hopwood J. Human alpha-L-iduronidase. CDNA isolation and expression. Proc Natl Acad Sci. 1991;88:9695-9.

4. Berget SM. Exon recognition in vertebrate splicing. J Biol Chem. 1995;270: 2411-4.

5. Varon R, Dutrannoy V, Weikert G, Tanzarella C, Antoccia A, Stöckl L, Spadoni E, Krüger LA, di Masi A, Sperling K. Mild Nijmegen breakage syndrome phenotype due to alternative splicing. Hum Mol Genet. 2006;15:679-89.

6. Stone J. Urine analysis in the diagnosis of mucopolysaccharide disorders. Ann Clin Biochem. 1998;35:207-25.

7. Hopwood J, Muller V. Biochemical discrimination of Hurler and Scheie syndromes. Clin Sci. 1979;57:265-72.

8. Maniatis T, Fritsch EF, Sambrook J. Molecular cloning: a laboratory manual: Cold spring harbor laboratory. NY: Cold Spring Harbor; 1982.

9. Mount SM. A catalogue of splice junction sequences. Nucleic Acids Res. 1982;10:459-72.

10. Skjørringe T, Tümer $Z$, Møller LB. Splice site mutations in the ATP7A gene. PLoS One. 2011;6:e18599. 
11. Chkioua L, Khedhiri S, Kassab A, Bibi A, Ferchichi S, Froissart R, Vianey-Saban C, Laradi S, Miled A. Molecular analysis of mucopolysaccharidosis type I in Tunisia: identification of novel mutation and eight novel polymorphisms. Diagn Pathol. 2011;6:39.

12. Chkioua L, Khedhiri S, Turkia HB, Chahed H, Ferchichi S, Dridi MFB, Laradi S, Miled A. Hurler disease (mucopolysaccharidosis type $\mathrm{H}$ ): clinical features and consanguinity in Tunisian population. Diagn Pathol. 2011;6:113.

13. Chkioua L, Khedhiri S, Turkia HB, Tcheng R, Froissart R, Chahed H, Ferchichi S, Dridi MFB, Vianey-Saban C, Laradi S. Mucopolysaccharidosis type I: molecular characteristics of two novel alpha-L-iduronidase mutations in Tunisian patients. Diagn Pathol. 2011;6:47.

14. Laradi S, Tukel T, Erazo M, Shabbeer J, Chkioua L, Khedhiri S, Ferchichi S, Chaabouni M, Miled A, Desnick R. Mucopolysaccharidosis I: a-L-Iduronidase mutations in three Tunisian families. J Inherit Metab Dis. 2005;28:1019-26.

15. Venturi N, Rovelli A, Parini R, Menni F, Brambillasca F, Bertagnolio F, Uziel G, Gatti R, Filocamo M, Donati M. Molecular analysis of 30 mucopolysaccharidosis type I patients: evaluation of the mutational spectrum in Italian population and identification of 13 novel mutations. Hum Mutat. 2002;20:231.

16. Clarke LA, Nelson PV, Warrington CL, Morris CP, Hopwood JJ, Scott HS Mutation analysis of 19 North American mucopolysaccharidosis type I patients: identification of two additional frequent mutations. Hum Mutat. 1994;3:275-82.

17. Cooper DN, Youssoufian H. The CpG dinucleotide and human genetic disease. Hum Genet. 1988;78:151-5.

Ready to submit your research? Choose BMC and benefit from:

- fast, convenient online submission

- thorough peer review by experienced researchers in your field

- rapid publication on acceptance

- support for research data, including large and complex data types

- gold Open Access which fosters wider collaboration and increased citations

- maximum visibility for your research: over $100 \mathrm{M}$ website views per year

At BMC, research is always in progress.

Learn more biomedcentral.com/submissions 